\title{
Thoracic endometriosis syndrome
}

\author{
D Guidozzi, MB BCh; E Rockson, MB BCh, MMed (Wits), FCOG (SA) \\ Department of Obstetrics and Gynaecology, Faculty of Health Sciences, University of the Witwatersrand, Johannesburg, South Africa
}

Corresponding author: D Guidozzi (deeguidozzi@gmail.com)

\begin{abstract}
A 35-year-old nulliparous patient with secondary infertility presented to a tertiary hospital in Johannesburg with abnormal uterine bleeding, and a history of two previous right-sided haemopneumothoraces. The second episode had been related to her menstruation, and required the insertion of an intercostal drain in order to treat the large haemothorax. A pleural biopsy revealed pleural endometriosis. She was treated with dienogest (Visanne) on discharge, and was awaiting follow-up with the cardiothoracic surgeons for continued and definitive management.
\end{abstract}

S Afr J Obstet Gynae 2018;24(3): 72-73. DOI:10.7196/SAJOG.2018.v24i3.1340

Thoracic endometriosis syndrome is a rare disease, commonly underdiagnosed and often inadequately treated, with a recurrence rate of $\sim 30 \%$, according to the British Thoracic Society guideline for management of spontaneous pneumothorax. ${ }^{[1,2]}$ It is not mentioned in the 2010 guidelines of the American College of Chest Physicians for the management of spontaneous pneumothorax, ${ }^{[1]}$ and South Africa does not have guidelines regarding the treatment of the condition. As a result, a high level of suspicion need to be maintained in women of childbearing age with recurrent haemoor pneumothoraces, to make the diagnosis. A multidisciplinary approach between cardiothoracic surgeons and gynaecologists is necessary for early disease recognition and adequate surgical and hormonal therapy to improve patient outcomes. ${ }^{[1,3]}$

\section{Case presentation}

A 35-year-old HIV-negative patient who had had one previous terminated pregnancy presented with a 2-week history of vaginal bleeding, complicated by symptomatic anaemia. She had previously been diagnosed with a multifibroid uterus in 2012, but had declined surgery, as she was concerned that it would result in a hysterectomy, and she wanted to remain fertile. A cytological smear of her cervix had been performed in 2012, and was said to be normal.

A month prior to this presentation, she had been admitted to the respiratory unit with an acute onset of shortness of breath. A diagnosis of spontaneous right-sided haemothorax was made, for which a chest drain had been inserted, draining $\sim 1 \mathrm{~L}$ of blood. On further questioning, it was established that a similar event had happened in 2015, but that no medical cause had been found at the time. The current episode was related to her menstruation.

She was a non-smoker, and an autoimmune screen and tuberculosis work-up had been negative on this admission. A computed tomography scan of her chest had shown normal lung parenchyma. During this admission, a pleural biopsy was performed by the cardiothoracic surgeons.

Pleural histology showed occasional strips of columnar epithelium, with ciliated metaplasia on the surface of the pleura. The epithelium was surrounded by endometrial-type stroma with associated interstitial haemorrhage. The focal endometrial stroma stained strongly positive for CD10. The conclusion was a finding of pleural endometriosis.

\section{Case management: Treatment and follow-up}

The patient's treatment was two-fold. Dienogest (Visanne) $2 \mathrm{mg}$ daily was initiated in order to ameliorate the symptoms of her endometriosis. In addition to her regular gynaecological follow-up, review by the cardiothoracic surgeons was planned, as she would benefit from more definitive surgical intervention in order to reduce her risk of recurrent haemopneumothoraces. Surgery could also aid in limiting her need for long-term hormonal treatment, allowing her a greater chance of conception.

Additionally, the problem of her multifibroid uterus and heavy menstrual bleeding was addressed. The patient was found to have a large prolapsed vaginal fibroid that was twisted and avulsed. Histology confirmed a benign leiomyoma. Two units of blood were transfused for her symptomatic anaemia, and she was given $1 \mathrm{~g}$ tranexamic acid 8-hourly for 3 days, to stop her vaginal bleeding. Her preference was for minimal surgical intervention, as she still wanted to remain fertile. Ultrasound confirmed no other significant submucosal fibroids. The patient was lost to follow-up, and did not attend her gynaecological or cardiothoracic appointments. In subsequent contact, by email, she reported remaining symptom-free, and did not wish for any further medical assessment or intervention.

\section{Discussion}

Thoracic endometriosis is rare, but is the most common site for endometriosis outside of the pelvis. ${ }^{[4]}$ Thoracic endometriosis syndrome refers to the clinical manifestations of the cyclical changes associated with the ectopic endometrial tissue, most frequently catamenial (menstrual) pneumothorax, followed by catamenial haemothorax, catamenial haemoptysis and endometriotic pulmonary nodules, in descending order of frequency. ${ }^{[4]}$ The word 'catamenial', meaning monthly, is derived from the Greek. ${ }^{[4]}$ Episodes generally occur from 24 hours before to 72 hours after the onset of menses, and tend to be recurrent. ${ }^{[4]}$ Chest pain is the most common symptom ( $\sim 90 \%$ of cases) followed by dyspnoea. ${ }^{[4]}$ Catamenial pneumothorax has an incidence of $3-6 \%$ in women with 
spontaneous pneumothoraces, and is right sided in the vast majority of cases (95\%). ${ }^{[4,5]}$ The British Thoracic Society guideline suggests that it may be as high as $25 \%$ in women who have undergone routine surgical treatment for recurrent pneumothorax. ${ }^{[5]}$ The mean age at presentation is $33-35$ years. ${ }^{[4]}$ Medical history that may suggest the diagnosis includes previous spontaneous pneumothorax or haemothorax, infertility, previous uterine surgery or endometriosis. ${ }^{[3]}$

Thoracic endometriosis is confirmed histologically if both endometrial stroma and glands are found in the specimen, and is considered probable if only endometrial stroma is seen. ${ }^{[4]}$ Histology should be interpreted in the context of the patient's history and clinical presentation. Immunohistochemical staining for oestrogen and progestogen receptors found on endometrial glands and stroma may aid the diagnosis. ${ }^{[1]} \mathrm{CD} 10$ antigen is found on endometrial stromal cells, and can help with identification of ectopic endometrial tissue even when endometrial epithelial cells are not present. ${ }^{[1]}$ Iron staining can identify haemosiderin-laden macrophages, which are a useful complementary marker. ${ }^{[1]}$

The pathophysiology behind the mechanism for the deposition of ectopic thoracic endometrial tissue remains poorly understood, but theories suggested include coelomic metaplasia of the pleura, lymphatic or vascular embolisation of endometrial tissue or retrograde menstruation. ${ }^{[4]}$ In addition, four main hypotheses for the development of catamenial pneumothoraces have been suggested. These include spontaneous rupture of subpleural blebs, necrosis of implants on visceral pleura with resultant air leakage, prostaglandininduced bronchiolar constriction or bronchial obstruction by endometrial implants, resulting in alveolar rupture, and finally, leakage of air from the genital tract through congenital or acquired defects in the diaphragm. ${ }^{[4-6]}$ The goal of treatment is to suppress or eradicate thoracic ectopic endometrial tissue, and prevent leakage of air across diaphragmatic defects. ${ }^{[4]}$ Surgical treatment, most commonly through the use of video-assisted thoracoscopic surgery (VATS), is the treatment of choice. ${ }^{[3,4]}$ Using VATS, the lung, parietal pleura and diaphragm can be inspected, and tissue samples taken. ${ }^{[3,4]}$ Excision of endometrial deposits, pleurodesis, pleural resection, lung wedge resection or diaphragmatic repair may be performed in order to facilitate treatment. ${ }^{[4]}$ Medical therapy alone is considered insufficient to treat the condition, as there is a high recurrence rate, and so it is viewed rather as an adjunct to surgical treatment, ${ }^{[3,4]}$ although medical treatment appears to have been effective in the case presently discussed. Hormones that mimic anovulation or menopause, including progestins, oral contraceptives, gonadotropin-releasing hormone $(\mathrm{GnRH})$ analogues or aromatase inhibitors, may be used. ${ }^{[4]} \mathrm{GnRH}$ analogues are especially useful for 6 - 12 months post-surgery, to suppress ectopic endometrial tissue while pleural adhesions form to allow for effective pleurodesis. ${ }^{[3]}$ It has been suggested that on average, longer periods of $\sim 17.5$ months of hormonal therapy are required after repeat surgeries. ${ }^{[4]}$ Recurrence is the most common complication of treatment, occurring in $8-40 \%$ of patients at a follow-up of 4 years. ${ }^{[2]}$

\section{Conclusion}

A high level of suspicion is needed to make this diagnosis in any woman of reproductive age with a spontaneous haemo-/ pneumothorax so that the opportunity for treatment is not missed. The ideal treatment is a combination of both medical and surgical therapy to maximise positive outcomes and reduce the risk of recurrence. As a result, a multidisciplinary approach to care involving the cardiothoracic surgeons and gynaecologists is necessary.

Acknowledgements. None.

Author contributions. Equal contributions.

Funding. None.

Conflicts of interest. None.

1. Legras A, Mansuet-Lupo A, Rousset-Jablonski C, et al. Pneumothorax in women of child-bearing age. Chest 2014;145(2):354-360. https://doi.org/10.1378\%2Fchest.13-1284

2. MacDuff A, Arnold A, Harvey J. Management of spontaneous pneumothorax: British Thoracic Society pleural disease guideline 2010. Thorax 2010;65(Suppl 2):ii18-ii31. https://doi. org/10.1136\%2Fthx.2010.136986

3. Visouli AN, Zarogoulidis K, Kougioumtzi I, et al. Catamenial pneumothorax. J Thorac Dis 2014;6(Suppl 4):S448-S460

4. Albores J, Fishbein G, Bando J. A 34-year-old woman with recurrent right-sided chest pain and dyspnoea. Chest 2015;148(5):el48-e151. https://doi.org/10.1378\%2Fchest.15-0813

5. Alifano M, Roth T, Broët SC, Schussler O, Magdeleinat P, Regnard J-F. Catamenial pneumothorax. Chest 2003;124(3):1004-1008

6. Gray R, Cormier M, Yedlicka J, Moncada R. Catamenial pneumothorax: Case report and literature review. J Thorac Imaging 1987;2(2):72-75. https://doi.org/10.1097\%2F00005382-198704000-00014

Accepted 12 November 2018. 